Portland State University

PDXScholar

$1-1-1986$

\title{
An Approximate Variational Method for Improved Thermodynamics of Molecular Fluids
}

\author{
M. S. Shaw \\ Los Alamos National Laboratory \\ J. D. Johnson \\ Los Alamos National Laboratory \\ John D. Ramshaw \\ Portland State University, jdramshaw@yahoo.com
}

Follow this and additional works at: https://pdxscholar.library.pdx.edu/phy_fac

Part of the Physics Commons

Let us know how access to this document benefits you.

\section{Citation Details}

M.S. Shaw, J.D. Johnson, and J.D. Ramshaw, "An approximate variational method for improved thermodynamics of molecular fluids," J. Chem. Phys. 45, 295 (1986)

This Article is brought to you for free and open access. It has been accepted for inclusion in Physics Faculty Publications and Presentations by an authorized administrator of PDXScholar. Please contact us if we can make this document more accessible: pdxscholar@pdx.edu. 


\title{
An approximate variational method for improved thermodynamics of molecular fluids
}

\author{
M. S. Shaw, J. D. Johnson, and J. D. Ramshaw \\ Theoretical Division, Los Alamos National Laboratory, Los Alamos, New Mexico 87545
}

(Received 1 October 1985; accepted 11 December 1985)

\begin{abstract}
For a certain class of thermodynamic perturbation theories, a generalization of the GibbsBogoliubov inequality holds through second order of perturbation theory and for a subset of terms the inequality is true to infinite order. Using this approximate variational principle, a perturbation theory is chosen for which the Helmholtz free energy of the reference system is minimized under the constraint that the first order term is identically zero. We apply these ideas to the determination of effective spherical potentials that accurately reproduce the thermodynamics of nonspherical molecular potentials. For a diatomic-Lennard-Jones (DLJ) potential with $l / \sigma=0.793$, the resulting spherical reference potential is identical to the median average over angles for the repulsive part of the potential, but differs in the attractive well. The variational effective spherical potential leads to more accurate thermodynamics than the median, however, particularly in the triple point region.
\end{abstract}

\section{INTRODUCTION}

The Gibbs-Bogoliubov inequality ${ }^{1-10}$ gives a rigorous upper bound to the Helmholtz free energy of one system in terms of the properties of a reference system. It has been used in practice by finding the lowest upper bound from a class of reference systems that are well described by available methods, e.g., the hard sphere system ${ }^{11,12}$ and the soft sphere system. ${ }^{13,14}$ Recent work by Goldman and Kumar ${ }^{15}$ has been directed toward using the rigorous Gibbs-Bogoliubov inequality in an approximate manner to choose a thermodynamic perturbation theory. We have taken a different approach whereby an approximate inequality is derived and implemented as though it were rigorous. The effective spherical potential thereby obtained is surprisingly accurate in reproducing the thermodynamics of the diatomic-Lennard-Jones (DLJ) potential with $l^{*}=l / \sigma=0.793$, where $l$ * is the reduced bond length between the two $\mathrm{LJ}$ centers on each molecule.

\section{VARIATIONAL METHOD}

Thermodynamic perturbation theories arise from choosing a parametric path in a single variable from a reference potential $\phi_{0}$ to the potential of interest $\phi$, and expanding the Helmholtz free energy as a Taylor series in that parameter. Because of numerical complications and limited knowledge of the reference system, the Taylor series is usually truncated after first or second order. Therefore, the choice of $\phi_{0}$ and the path of $\phi$ strongly influence the accuracy of the truncated Taylor series.

In previous work ${ }^{16-19}$ we have used a formulation of general perturbation theories based on Smith $e t$ al ${ }^{20}$ and have restricted the class of perturbation theories considered. One can characterize this class of perturbation theories by a function $R$ which determines the path by

$$
R\left(\phi_{\gamma}-\phi_{0}\right)=\gamma R\left(\phi-\phi_{0}\right) \text {. }
$$

Here $R$ is any odd invertible function, $\gamma$ is the path parameter, and $\phi_{\gamma}$ is the potential along the path from $\phi_{0}$ to $\phi$ at the parameter value of $\gamma$. Note that at $\gamma=0, \phi_{\gamma}=\phi_{0}$, and at $\gamma=1, \phi_{\gamma}=\phi$. The choice $R(x)=x$ gives the familiar $\lambda$ expansion. ${ }^{21}$ One can easily determine $\phi_{\gamma}$ and its derivatives as

$$
\phi_{r}=\phi_{0}+R^{-1}\left[\gamma R\left(\phi-\phi_{0}\right)\right]
$$

and

$$
\left.\left.\frac{d^{n} \phi_{\gamma}}{d \gamma^{n}}\right)_{\gamma=0}=\frac{d^{n} R^{-1}(x)}{d x^{n}}\right)_{x=0}\left[R\left(\phi-\phi_{0}\right)\right]^{n} .
$$

Since $R$ is odd, all even derivatives of $\phi_{\gamma}$ are zero at $\gamma=0$. Then we can write

$$
\phi_{\gamma}-\phi_{0}=\gamma \phi^{(1)}+\frac{\gamma^{3}}{3 !} \phi^{(3)}+\frac{\gamma^{5}}{5 !} \phi^{(5)}+\cdots,
$$

where $\phi^{(n)}$ denotes $\left.d^{n} \phi_{\gamma} / d \gamma^{n}\right)_{\gamma=0}$. For a two-body potential, the total energy of a configuration of particles is $U=\frac{1}{2} \Sigma_{i \neq j} \phi_{i j}$, and consequently

$$
U_{\gamma}-U_{0}=\gamma U^{(1)}+\frac{\gamma^{3}}{3 !} U^{(3)}+\cdots,
$$

where $U^{(n)} \equiv \frac{1}{2} \Sigma_{i \neq j} \phi_{i j}^{(n)}$.

One can easily show (see, e.g., Mansoori and Canfield $^{12}$ ) that the ratio of partition functions for the two systems may be written as

$$
Q_{r} / Q_{0}=\left\langle\exp \left[-\beta\left(U_{r}-U_{0}\right)\right]\right\rangle_{0},
$$

where \langle\rangle$_{0}$ represents the expectation value over the probability distribution function in the reference system. If $Q_{r} / Q_{0}>1$, then the corresponding Helmholtz free energies, $A=-k T \ln Q$, have the property that $A_{\gamma}<A_{0}$. By writing the exponential as a Taylor series and inserting Eq. (5), we have

$$
\begin{aligned}
\frac{Q_{\gamma}}{Q_{0}}=\langle 1 & -\beta\left(\gamma U^{(1)}+\frac{\gamma^{3}}{3 !} U^{(3)}+\cdots\right) \\
& +\frac{\beta^{2}}{2 !}\left(\gamma U^{(1)}+\frac{\gamma^{3}}{3 !} U^{(3)}+\cdots\right)^{2} \\
& -\frac{\beta^{3}}{3 !}\left(\gamma U^{(1)}+\frac{\gamma^{3}}{3 !} U^{(3)}+\cdots\right)^{3} \\
& +\cdots\rangle_{0} .
\end{aligned}
$$


One can collect the terms involving only $\left(\gamma U^{(1)}\right)^{n}$ and sum them to get $\exp \left(-\beta \gamma U^{(1)}\right)$, and the first terms that are ignored are third order in the perturbation theory. So we have

$$
Q_{\gamma} / Q_{0}=\left\langle\exp \left(-\beta \gamma U^{(1)}\right)+O\left(\gamma^{3}\right)\right\rangle_{0} .
$$

If the terms $\left\langle O\left(\gamma^{3}\right)\right\rangle_{0}$ are small and we use a special case of the Jensen inequality ${ }^{22}\left\langle e^{x}\right\rangle \geqslant e^{\langle x\rangle}$, then for $\gamma=1$ we get

$$
A \leqslant A_{0}+\left\langle U^{(1)}\right\rangle_{0} \text {. }
$$

For $R(x)=x$, the terms $O\left(\gamma^{3}\right)$ are identically zero and we recover the Gibbs-Bogoliubov inequaltiy. In fact, one can prove the Gibbs-Bogoliubov inequality by applying the Jensen inequality directly to Eq. (6). For this more general class of perturbation theories, the inequality is approximate with some third and higher order contributions neglected. The extra freedom from the choice of $R$ allows us to find upper bounds that are lower than with the Gibbs-Bogoliubov inequality provided the neglect of the terms $O\left(\gamma^{3}\right)$ is a good approximation.

As an aside, it may be helpful at this point to compare the terms in Eq. (7) with the more standard perturbation expansion of excess Helmholtz free energy $A^{\text {ex }}$. We will show that the terms $\left\langle O\left(\gamma^{3}\right)\right\rangle_{0}$ which we dropped to get Eq. (9) are also of third and higher order in the general Smith et al. ${ }^{20}$ formulation for $A^{\text {ex }}$. From the definition

$$
A_{\gamma}^{\mathrm{ex}}=-(1 / \beta) \ln Q_{\gamma}^{\mathrm{ex}}
$$

Eq. (7), and the fact that the ideal contribution to $Q$ is independent of $\gamma$, we have

$$
\left.\left.\frac{\partial A_{\gamma}^{\mathrm{ex}}}{\partial \gamma}\right)_{\gamma=0}=-\frac{1}{\beta} \frac{1}{Q_{0}} \frac{\partial Q_{\gamma}}{\partial \gamma}\right)_{\gamma=0}=\left\langle U^{(1)}\right\rangle_{0} .
$$

That is, the first order term in $A^{\text {ex }}$ comes from the first order term in $Q / Q_{0}$. By the $n$th order term in $A^{\text {ex }}$, we mean the term

$$
\left.\frac{1}{n !} \frac{\partial^{n} A_{\gamma}^{\mathrm{ex}}}{\partial \gamma^{n}}\right)_{\gamma=0}
$$

in the Taylor expansion of $A_{r}^{\text {ex }}$,

$$
\left.\left.A_{\gamma=1}^{\mathrm{ex}}=A_{0}^{\mathrm{ex}}+\frac{\partial A_{\gamma}^{\mathrm{ex}}}{\partial \gamma}\right)_{\gamma=0}+\frac{1}{2 !} \frac{\partial^{2} A^{\mathrm{ex}}}{\partial \gamma^{2}}\right)_{\gamma=0}+\cdots
$$

and similarly for $Q / Q_{0}$. This is in accordance with the usual terminology for the order of a term in a perturbation expansion of the excess Helmholtz free energy. The second order term is given by

$$
\begin{aligned}
\left.\frac{1 \partial^{2} A_{\gamma}^{\text {ex }}}{2 \partial \gamma^{2}}\right)_{\gamma=0} & =-\frac{1}{2 \beta}\left[\frac{1}{Q_{0}} \frac{\partial^{2} Q_{\gamma}}{\partial \gamma^{2}}-\left(\frac{1}{Q_{0}} \frac{\partial Q_{\gamma}}{\partial \gamma}\right)^{2}\right]_{\gamma=0} \\
& =\frac{1}{2} \beta\left[-\left\langle\left(U^{(1)}\right)^{2}\right\rangle_{0}+\left(\left\langle U^{(1)}\right\rangle_{0}\right)^{2}\right] .
\end{aligned}
$$

The second order term in $A^{\text {ex }}$ has contributions from the first and second order terms in $Q / Q_{0}$. In general, the $n$th order term in $A^{\text {ex }}$ will be a function of the first through $n$th order terms in $Q / Q_{0}$.

We now return to the main development. We will restrict our study to those cases where $U_{0}$ and $R$ are related in such a way that $\left\langle U^{(1)}\right\rangle_{0}=0$, and therefore $A \leqslant A_{0}$. We further specialize to the case of effective spherical potentials for molecular systems. The choice $\left\langle U^{(1)}\right\rangle_{0}=0$ is numerical- ly convenient in that a spherical $\phi_{0}$ may be chosen from the condition $^{17}$

$$
\int R\left[\phi(r, \Omega)-\phi_{0}(r)\right] d \Omega=0 .
$$

Then $\left\langle U^{(1)}\right\rangle_{0}$ becomes zero and $A_{0}$ is evaluated for a spherical potential, for which many accurate methods are available. Also, by choosing the first order term to be zero, one hopes that the higher order terms would be small, which is essential for Eq. (9) to be useful.

\section{GENERALIZATION TO $r$ DEPENDENT PERTURBATION THEORIES}

One usually considers perturbation theories in which $R$ is a simple function of one variable. However, there is no necessity for such a restriction on $R$. All that is required is that a parametric path from $\phi_{0}$ to $\phi$ be specified. We will consider here a choice of $R$ that also depends on the center of mass separation $r$ of a nonspherical potential $\phi(r, \Omega)$. That is, for each $r, \phi_{r}$ is chosen from

$R\left[\phi_{r}(r, \Omega)-\phi_{0}(r) ; r\right]=\gamma R\left[\phi(r, \Omega)-\phi_{0}(r) ; r\right]$.

Note that we still have a well-defined path from $\phi_{0}(r)$ to $\phi(r, \Omega)$ as a function of $\gamma$.

The reason for choosing this form is to permit the determination of the lowest allowed $\phi_{0}(r)$ at each value of $r$. If we choose two potentials $\phi_{A}(r)$ and $\phi_{B}(r)$ such that $\phi_{A}(r) \geqslant \phi_{B}(r)$ for all $r$, then for an arbitrary configuration of particles

$$
U_{A}=\frac{1}{2} \sum_{i \neq j} \phi_{A}\left(r_{i j}\right) \geqslant U_{B}=\frac{1}{2} \sum_{i \neq j} \phi_{B}\left(r_{i j}\right) .
$$

From the definition of the partition function, we then have that $Q_{A} \leqslant Q_{B}$ and consequently $A_{A} \geqslant A_{B}$. Therefore, the lowest choice of $\phi_{0}(r)$ allowed by Eq. (13) gives for this class of perturbation theories the best choice of $A_{0}$, provided the error in Eq. (9) is small.

The general procedure may therefore be summarized as follows. We require that $\phi_{0}$ and $R$ are related by Eq. (13), so that $\left\langle U^{(1)}\right\rangle_{0}=0$ and Eq. (9) reduces to $A \leq A_{0}$. We then minimize $A_{0}$ with respect to the remaining freedom in $\phi_{0}$ and $R$, which is equivalent to minimizing $\phi_{0}(r)$ itself at each point $r$ subject to the constraint of Eq. (13). This minimization simultaneously determines $\phi_{0}$ and $R$, but only the former is of interest so the latter is not explicitly determined.

The development by which we have arrived at this procedure is totally dependent on the neglect of terms which cannot be readily calculated. For sufficiently small anisotropy, these terms will also be small. Lacking a criterion for what is "sufficiently small," there is no a priori basis for expecting it to work as well as it does. The real justification for the procedure lies in the accuracy of the results to which it leads; see Sec. V.

\section{DETERMINATION OF THE BEST $\phi_{0}(r)$}

Now that we have a general procedure, it is necessary to find an efficient numerical method for the determination of the lowest allowed $\phi_{0}(r)$. We want to minimize $\phi_{0}(r)$ at each $r$ subject to the constraint of Eq. (13) with $R$ in the class of 
perturbation theories giving Eq. (9), i.e., $R$ an odd invertible function or the limit of such. We can rewrite Eq. (13) in terms of the probability density

$$
\rho(E)=\int \delta[\phi(r, \Omega)-E] d \Omega / \int d \Omega
$$

of an orientation having potential energy $E$ at a given value of $r$ :

$$
\int_{-\infty}^{\infty} \rho(E) R\left(E-E_{0}\right) d E=0,
$$

where $E_{0}$ is just $\phi_{0}(r)$ for the given value of intermolecular separation $r$. We then look for the function $R$ that leads to the lowest value of $E_{0}$. Conversely, we may determine the lowest value of $E_{0}$ for which a nontrivial function $R$ satisfying Eq. (15) exists, and this is how we actually proceed in practice. Integrating by parts, we obtain

$\left.s(E) R\left(E-E_{0}\right)\right|_{-\infty} ^{\infty}-\int_{-\infty}^{\infty} s(E) R^{\prime}\left(E-E_{0}\right) d E=0$,

where

$$
s(E)=\int_{-\infty}^{E} \rho(x) d x .
$$

From the definition of $\rho(E), s(E)$ is the cumulative distribution function; i.e., the fraction of orientations $\Omega$ with $\phi(r, \Omega)<E$ for a given $r$. Clearly, $s(x)=0$ for $x<a$ and $s(x)=1$ for $x>b$, where $a$ is the lowest value of $\phi(r, \Omega)$ and $b$ is the highest value of $\phi(r, \Omega)$ at the given value of $r$. Equation (16) can be rewritten as

$$
\int_{0}^{\infty} R^{\prime}(y)\left\{1-\left[s\left(E_{0}+y\right)+s\left(E_{0}-y\right)\right]\right\} d y=0,
$$

since $R(0)=0$ from our restriction to odd functions. We note that $R^{\prime}(y)>0$ for all $y$ because $R$ is odd and invertible. Now define the function

$$
f\left(y, E_{0}\right)=1-\left[s\left(E_{0}+y\right)+s\left(E_{0}-y\right)\right],
$$

which is determined solely by the probability density $\rho(E)$. Note that $f\left(y, E_{0}\right)=0$ for $y>\max \left(b-E_{0}, E_{0}-a\right)$. Now if $E_{0}$ is too small, then $f\left(y, E_{0}\right)$ is strictly positive for $0 \leqslant y<\max \left(b-E_{0}, E_{0}-a\right)$, and it is then impossible to satisfy Eq. (18) with a nontrivial $R(y)$. For example, let $E_{0}=a$ and $b \neq a$. Then $s\left(E_{0}-y\right)=0$ for all $y>0$. For the interval $0 \leqslant y<b-a, s\left(E_{0}+y\right)<1$ and therefore $f\left(y, E_{0}\right)>0$ for the same interval. Also, note that $f\left(y, E_{0}\right)$ is monotonically decreasing with $E_{0}$ for fixed $y$, because $s(x)$ is monotonically increasing. In order for Eq. (18) to be satisfied, $f\left(y, E_{0}\right)$ must clearly be zero somewhere in the interval $0 \leqslant y<\max \left(b-E_{0}, E_{0}-a\right)$. There will be some critical value of $E_{0}$, such that $f\left(y, E_{0}\right)$ is zero at one or more values of $y$ and positive elsewhere in this interval. This value of $E_{0}$ depends only on the function $s(y)$ which in turn depends on $r$. It is the smallest $E_{0}$ for which Eq. (18) can be satisfied, and thus just what we wish to determine. For values of $E_{0}$ slightly larger than this critical value, the required $R^{\prime}$ will be sharply peaked in the vicinity of a small region of slightly negative $f$. In the limit as $E_{0}$ approaches its minimum value from above, $R^{\prime}(y)$ approaches a delta function and $R(y)$ becomes a step function, with the step occurring at the point in the interval $0<y<\max \left(b-E_{0}, E_{0}-a\right)$ where $f\left(y, E_{0}\right)$ just touches zero.

Thus, the minimum $E_{0}$ for which Eq. (18) can be satisfied is simply the smallest value of $E_{0}$ for which $f\left(y, E_{0}\right)$ goes to zero at some point in the range $0<y<\max (b$ $\left.-E_{0}, E_{0}-a\right)$. To find this $E_{0}$ we first invert the function $s(y)$ to obtain $y(s)$, the value of the potential such that a fraction $s$ of the orientations are lower in energy. That is,

$$
\int \theta[y(s)-\phi(r, \Omega)] d \Omega / \int d \Omega=s,
$$

where $\theta(x)=1$ for $x \geqslant 0$ and $\theta(x)=0$ otherwise. Clearly then, $y(0)=a_{2} y(1)=b$, and $y(1 / 2)$ is the median. In terms of $y(s)$, the condition $f\left(y, E_{0}\right)=0$ becomes $E_{0}=\frac{1}{2}[y(s)+y(1-s)]$. We then simply vary $s$ from 0 to $1 / 2$; the smallest value of $\frac{1}{2}[y(s)+y(1-s)]$ encountered in doing so is the desired minimal $E_{0}$, which is in turn the optimal choice for $\phi_{0}(r)$ at that value of $r$. If the minimal $E_{0}$ occurs at $s=1 / 2$, then $\phi_{0}(r)$ is the median average over angles. If the minimal $E_{0}$ occurs at $s=0$, then $\phi_{0}(r)$ is the midpoint between $a$ and $b$. For the DLJ potential studied in the next section, we will see that for almost all values of $r$ one of these two values is the minimum.

In order to evaluate the required quantities numerically, we have constructed an approximate probability density at fixed $r$ from the weights $w_{i}$ and values $\epsilon_{i}$ used in a GaussLegendre quadrature in the angular coordinates. The set of $\left(w_{i}, \epsilon_{i}\right)$ was reordered in ascending order of $\epsilon_{i}$ 's. The probability density was taken to be piecewise constant with the value

$$
\frac{1}{2} \frac{\left(w_{i}+w_{i-1}\right)}{\epsilon_{i}-\epsilon_{i-1}} \text { for } \epsilon_{i-1}<\epsilon<\epsilon_{i} .
$$

This leads to an $s(y)$ such that

$$
s\left(\epsilon_{i}\right)=\frac{1}{2} w_{i}+\sum_{j=1}^{i-1} w_{j},
$$

with $s(y)$ for intermediate $y$ obtained by linear interpolation. A table of $y(s)$ was then constructed for regular increments in $s$ from 0 to 1 . For this study $\Delta s$ was taken to be 0.01 . Finally, $\phi_{0}(r)$ at $r$ was determined by the minimum value of $\frac{1}{2}[y(s)+y(1-s)]$ in the table. The order of quadrature used was systematically increased until the fluctuations in $E_{0}$ were significantly less than $1 \%$.

Thermodynamic quantities for $\phi_{0}(r)$ were calculated using the perturbation theory developed by Ross ${ }^{13}$ based on earlier work by Rasaiah and Stell ${ }^{11}$ and Mansoori and Canfield. ${ }^{12}$

\section{RESULTS}

For comparison with a simulation of anisotropic potentials we have chosen a DLJ potential with $l / \sigma=0.793$. This corresponds roughly to $\mathrm{CO}_{2}$ and is the same potential used in previous analytical work by MacGowan et $a l^{23,24}$ and in MD simulations by Singer et $a .^{25}$ For significantly smaller values of $l / \sigma$, the median ${ }^{16,26}$ gives good thermodynamics ${ }^{23}$ and will be nearly identical to our variational $\phi_{0}(r)$. Figure 1 shows a plot of $\frac{1}{2}[y(s)+y(1-s)]$ as a function of $s$ for values of $r$ where $s=1 / 2$ (the median) was minimal, where 


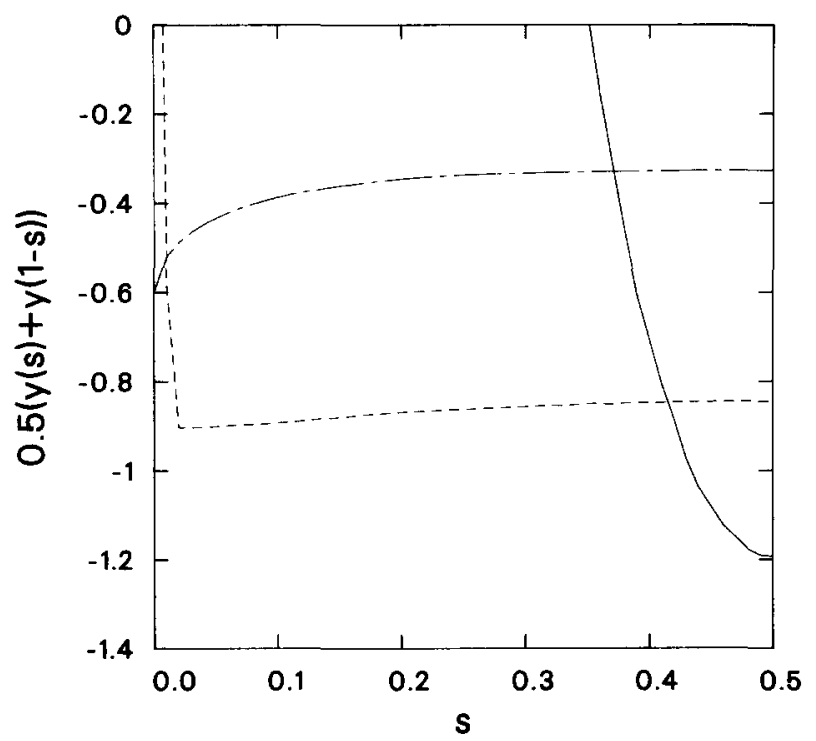

FIG. 1. The function $\{[y(s)+y(1-s)]$ is plotted vs $s$. The minimum value of the function is the variational choice for the potential. - is for $r^{*}=1.3$ and the median $(s=0.5)$ is minimum..-- is for $r^{*}=1.7$, and neither the median nor the midpoint is minimum..-- is for $r^{*}=2.0$ and the midpoint $(s=0.0)$ is minimum.

some intermediate $s$ was minimal, and where $s=0$ (the midpoint) was minimal.

Figure 2 shows the variational minimum potential $\phi_{0}(r)$ as well as the median, the midpoint, the unweighted average, and two extreme orientations in the attractive well. In the repulsive region, $\phi_{0}(r)$ is always the median for this potential and is therefore not shown. All quantities are shown in reduced units; $r^{*}=r / \sigma$ and $\phi^{*}\left(r^{*}\right)=\phi(r / \sigma) / \epsilon$.

Figure 3 compares the reduced pressure $P^{*}=P \sigma^{3} / \epsilon$ vs reduced density $\rho^{*}=\rho \sigma^{3}$ for $\phi_{0}(r)$, the median, and fits to

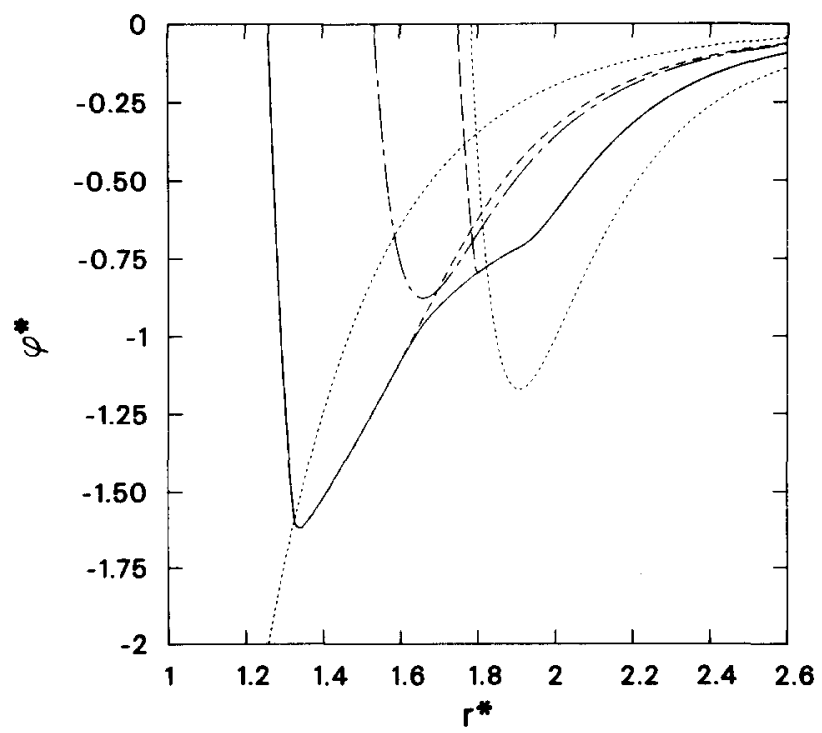

FIG. 2. Various potentials, $\phi^{*}$, are plotted vs the radius, $r^{*},-$ is the variational potential. - - is the potential median. - - - is the unweighted angular average. - - - is the midpoint. - . - is for two extreme orientations of the anisotropic potential. For $r * \$ 1.6$ the variational potential and the potential median are identical. For $r^{*} \gtrsim 1.8$ the variational potential and the midpoint are identical.

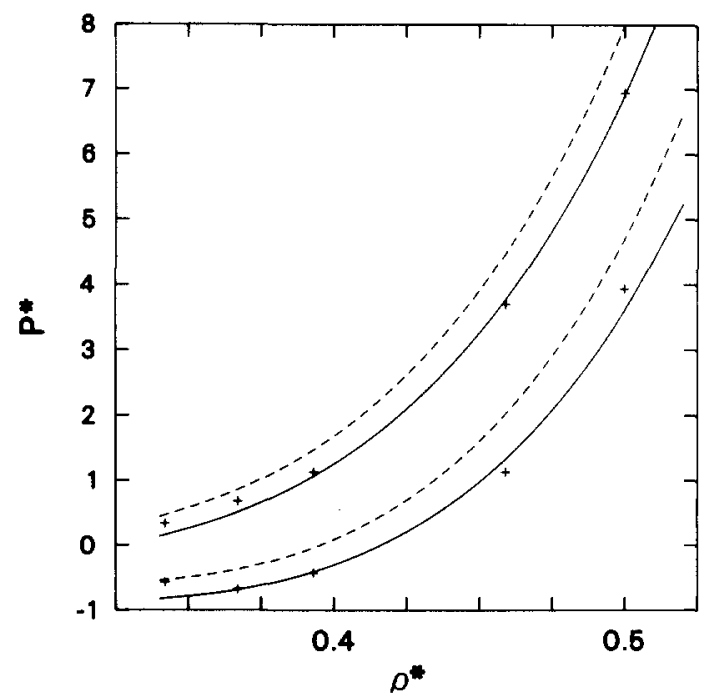

FIG. 3. Reduced pressure, $P *$, vs reduced density, $\rho^{*}$. — is calculated from the variational potential. . . - is from the potential median. + are from MD simulations as noted in the text. $T^{*}=1.9$ for the upper grouping and $T^{*}=1.3$ for the lower grouping.

the MD simulations of Singer et $a l_{.}{ }^{25}$ The reduced temperature $T^{*}=T / \epsilon$ is 1.9 for the upper grouping of calculations and 1.3 for the lower grouping. In Fig. 4 the reduced internal energy $E^{*}=E / N \epsilon=U^{*}+\frac{5}{3} T^{*}$ (where $U^{*}$ is the reduced configurational energy) is plotted vs $\rho^{*}$ for the same values of $T^{*}$. For $\rho^{*}=0.5$ and $T^{*}=1.9$, the accuracy of the fit to MD was questionable because this point was apparently outside the range of the data used for the fit. We have therefore recalculated it using MD as described in Johnson et al., ${ }^{17}$ with the results $P^{*}=6.94$ and $E^{*}-6.47$. The results for $\phi_{0}(r)$ agree with the DLJ simulations to about the accuracy of the Ross procedure itself. Some ad hoc modifications of the median by MacGowan ${ }^{24}$ lead to results for $E^{*}$ roughly 3 / 4 of the way from that of the median to that of $\phi_{0}(r)$. Calculations with a radial median ${ }^{18}$ lead to similar improvement over the median, but less accurate than that obtained by using $\phi_{0}(r)$.

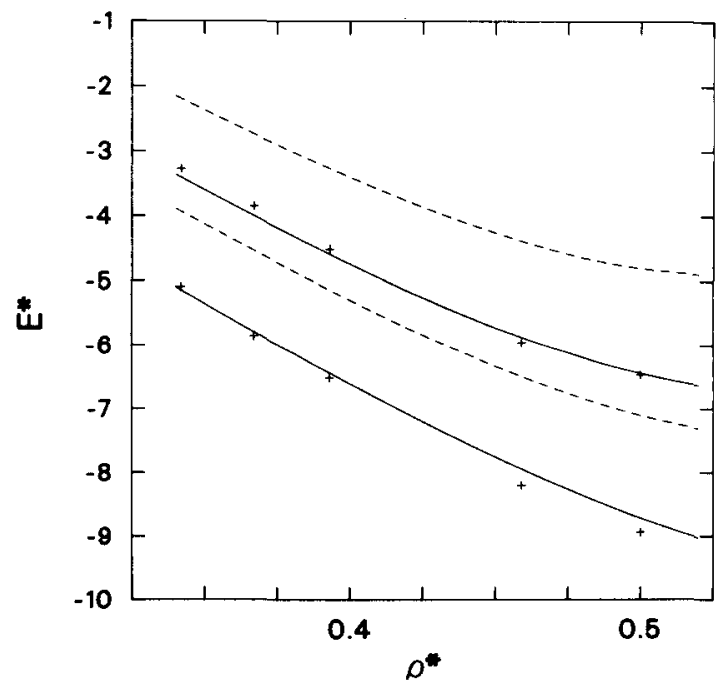

FIG. 4. Reduced energy, $E$ * vs reduced density, $\rho^{*}$. Symbols are the same as in Fig. 3. 


\section{CONCLUSION}

We have found an approximate variational method that leads to a significant improvement in accuracy over the median potential in the triple point region for a diatomic-Lennard-Jones potential with anisotropy comparable to $\mathrm{CO}_{2}$. The resulting effective spherical potential is identical to the median for the repulsive part of the potential, which dominates in high-density, high-temperature regions where the median itself is very accurate, ${ }^{16-18}$ but differs in the attractive well. It will be interesting to see whether this procedure continues to be accurate for different types of anisotropic potentials where the median does not give good results.

\section{ACKNOWLEDGMENT}

We wish to thank $\mathbf{R}$. LeSar for a careful reading of the manuscript and for a number of helpful suggestions regarding the presentation.

'J. P. Hansen and I, R. McDonald, Theory of Simple Liquids (Academic, London, 1976), p. 148.

${ }^{2}$ J. W. Gibbs, Elementary Principles in Statistical Mechanics (Scribner's, New York, 1902), Chap. XI, p. 129.

${ }^{3}$ R. Peierls, Phys. Rev. 54, 918 (1938).

${ }^{4} \mathrm{~N}$. N. Bogoliubov. A variational principle is attributed to Bogoliubov in Ref. 5 and is cited in the translation of Ref. 6 as follows: "N. N. Bogoliubov, see dissertation by I. A. Kvasnikov [in Russian] (V. A. Steklov Mathematical Inst., AN SSSR, 1958)." Note that in both Refs. 5 and 6 the papers were presented by Academician N. N. Bogoliubov.

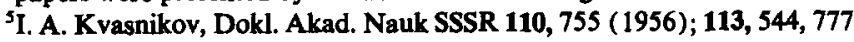
(1957).
${ }^{6}$ V. V. Tolmachev, Dokl. Akad. Nauk SSSR 134, 1324 (1960).

${ }^{7}$ M. Girardeau, J. Math. Phys. 3, 131 (1962).

${ }^{8}$ R. B. Griffiths, J. Math. Phys. 5, 1215 (1964).

${ }^{9}$ T. Lukes and R. Jones, J. Phys. A 1, 29 (1968).

${ }^{10}$ A. Isihara, J. Phys. A 1, 539 (1968).

"J. C. Rasaiah and G. Stell, Mol. Phys. 18, 249 (1970); G. Stell, Chem. Phys. Lett. 4, 651 (1970).

${ }^{12}$ G. A. Mansoori and F. B. Canfield, J. Chem. Phys. 51, 4958 (1969); 53, 1618 (1970).

${ }^{13}$ M. Ross, J. Chem. Phys. 71, 1567 (1979).

${ }^{14}$ D. A. Young and F. J. Rogers, J. Chem. Phys. 81, 2789 (1984).

${ }^{15}$ S. Goldman and B. Kumar, J. Chem. Phys. 82, 4276 (1985).

Note added in proof: See D. MacGowan, J. Chem. Phys. (to be published) for a comment pointing out a problem with Goldman and Kumar's method.

${ }^{16}$ M. S. Shaw, J. D. Johnson, and B. L. Holian, Phys. Rev. Lett. 50, 1141 (1983).

17J. D. Johnson, M. S. Shaw, and B. L. Holian, J. Chem. Phys. 80, 1279 (1984).

${ }^{18}$ J. D. Johnson and M. S. Shaw, J. Chem. Phys. 83, 1271 (1985).

${ }^{19}$ M. S. Shaw and J. D. Johnson, Proceedings of the Eighth Symposium (International) on Detonation (to be published).

${ }^{20}$ W. R. Smith, Can J. Phys. 52, 2022 (1974); W. R. Smith, I. Nezbeda, T. W. Melnyk, and D. D. Fitts, Faraday Discuss Chem. Soc. 66, 130 (1978).

${ }^{21}$ R. W. Zwanzig, J. Chem. Phys. 22, 1420 (1954).

${ }^{22}$ For convex functions $f$, the inequality given by $\sum_{i=1}^{n} \alpha_{f} f\left(x_{i}\right)>f\left(\sum_{i=1}^{n} \alpha_{i} x_{i}\right)$ is usually called the Jensen inequality. A convenient discussion may be found in $E$. F. Beckenbach and $R$. Bellman, Inequalities (Springer, Berlin, 1965), pp. 16-19. A special case of the inequality is attributed to M. Lüroth in M. Pringsheim, Sitzungsber. d. math. phys. Classe d. k. bayer. Akad. d. W., t. 32, pp. 163-192. Nachtrag ..., ibid pp. 295-303. This reference is given in J. L. W. V. Jensen, Acta Math. 30, 175 (1905), where the derivation of the inequality is presented.

${ }^{23}$ D. MacGowan, E. M. Waisman, J. L. Lebowitz, and J. K. Percus, J. Chem. Phys. 80, 2719 (1984).

${ }^{24}$ D. MacGowan, J. Chem. Phys. 81, 3224 (1984).

${ }^{25}$ K. Singer, A. Taylor, and J. V. L. Singer, Mol. Phys. 33, 1757 (1977).

${ }^{26}$ J. L. Lebowitz and J. K. Percus, J. Chem. Phys. 79, 443 (1983). 